\title{
Itinerarium, Netzwerk und Bewegung
}

Kehren wir an dieser Stelle noch einmal kurz zu unserer Einleitung zurück. Wir sollten uns nochmals verdeutlichen, dass die Reiseliteratur bis weit in die zweite Hälfte des 20. Jahrhunderts als weitgehend marginal galt und keinen hohen Stellenwert in der Gattungshierarchie einnahm. Man las sie in aller Regel faktenorientiert und dokumentarisch und versuchte, in erster Linie ihren Wirklichkeitsgehalt $\mathrm{zu}$ ergründen und ihre Wertigkeit daran auszurichten. Diese weitgehend marginale Position der Reiseliteratur hat sich in Verlauf der zurückliegenden Jahrzehnte grundlegend gewandelt. Die Reiseliteratur gehört heute zu jenen Schreibformen und Vermittlungsweisen, in denen sich am deutlichsten und dringlichsten die jeweiligen multi-, inter- und transkulturellen Beziehungsgeflechte ausloten lassen. Am Beispiel der Reiseliteratur werden die Herausforderungen und Grenzen, aber auch die Vorstellungen und Wünsche ihrer Autorinnen und Autoren wie ihrer Leserinnen und Leser ablesbar, die sich auf ihrer Reise gleichsam zwischen den Kulturen bewegen.

Am Beispiel der Reiseliteratur wollen wir ab der Frühen Neuzeit und bis zum gegenwärtigen Zeitpunkt die historischen und sozialen, politischen und ökonomischen, nicht zuletzt aber auch die kulturellen und schreibbezogenen Hintergründe ausleuchten, welche die Reiseliteratur als Teil der Literaturen der Welt geprägt haben. Dabei soll es um die hermeneutischen Bewegungen von Reisen und Schreiben, aber auch um die gattungsspezifischen Entwicklungen gehen, welche die Reiseliteratur über die Jahrhunderte ausgezeichnet haben. Denn auch wenn die Reiseliteratur lange Jahrhunderte hindurch für marginal gehalten wurde, so hat sie in ihrem Korpus doch ein Wissen aufbewahrt, dass wir uns durch ein genaues Studium aneignen können. Dieses Lebenswissen erscheint uns als ein Erlebenswissen (im Augenblick der Reise selbst), aber auch als ein Überlebenswissen und ein Zusammenlebenswissen, aus dem wir generelle Schlüsse für die jeweiligen Kulturen, vor allem aber auch für unsere eigene Kultur ziehen können. Denn die Reiseliteratur hält noch viele Einsichten in jene Verhaltensmuster bereit, mit denen wir dem Anderen in seiner allgemeinsten Form begegnen. Reiseliteratur ist in diesem $\mathrm{Zu}$ sammenhang eine Interdependenz von Bewegung und Begegnung - und genau aus dieser Kombination zieht sie ihren Reiz.

Unsere Ausgangshypothese dabei ist, dass uns die Reiseliteratur als ein ZwischenWeltenSchreiben sagt, wohin die Reise unserer Gesellschaften geht und welches die Herausforderungen sind, mit denen wir es im Verlauf der kommenden Jahrzehnte gerade im Beziehungsgeflecht der Kulturen zu tun bekommen

Ә Open Access. (C) 2020 Ottmar Ette, publiziert von De Gruyter. (cc) BY-NC-ND Dieses Werk ist lizenziert unter der Creative Commons Attribution-NonCommericial-NoDerivatives 4.0 Lizenz.

https://doi.org/10.1515/9783110650686-005 
werden. Es geht mir also auch um die prospektive, um die seismographische Funktion von Literatur und Reiseliteratur, die uns etwas anzeigen kann, von dem wir als Gesellschaft wenig wissen.

Um uns diese prospektive Form der Erkenntnis unserer eigenen Geschichte wie unserer eigenen Zukunft $\mathrm{zu}$ öffnen, ist es keineswegs notwendig, uns mit der jeweils neuesten Gegenwartsliteratur des Reisens zu beschäftigen; gewiss, wir werden dies an geeigneter Stelle im weiteren Verlauf der Vorlesung tun. Doch die Reiseliteratur gerade auch der Frühen Neuzeit enthält viele Elemente und auch Paradigmen, die uns Auskunft über unsere Geschichte, aber auch über unsere weiteren Entwicklungsmöglichkeiten sowie Grenzen geben. Dazu sollte unser Durchgang durch die Kartographie frühneuzeitlicher Kartenkunst schon einen ersten Vorgeschmack geben. Wir wenden uns der Vergangenheit zu, um etwas über unsere Zukunft zu erfahren.

Reiseliteratur ist gewiss eine Form des Schreibens, die etwas über den Reisevorgang selbst sowie die dabei in Augenschein genommenen Länder sagt. Und sie sagt uns nicht nur etwas über sich selbst aus, sondern selbstverständlich auch über die von ihr bereisten und besuchten Orte. Was sich hier so banal anhört, hat es eigentlich im Grunde in sich. Denn man könnte in gewisser Weise sagen, dass man Orte und Räume im Grunde nur wahrnehmen kann, wenn man auch ihre jeweiligen Bewegungen und die dort angesiedelten Motionen mitberücksichtigt, also nicht nur sieht, was sich dort befindet, sondern auch, was sich dort befand, was sich dort bewegt, was auf welche Weise in welche Bewegungsmuster eingebaut ist. Denn Räume sind im Grunde Bewegungs-Räume: Der Raum wird durch Bewegungen hergestellt, die ihn queren und kreuzen, die ihn ausspannen und traversieren.

Um dies zu verdeutlichen, möchte ich Ihnen gerne gleich $\mathrm{zu}$ Beginn einige kurze Überlegungen von Italo Calvino vorstellen, der 1974 in einem kurzen Essay über die ligurische Stadt Savona die folgenden Überlegungen anstellte:

\footnotetext{
Wenn man einen Ort beschreiben will, ihn vollständig beschreiben will, nicht als flüchtige Erscheinung, sondern als Teil eines Raumes, der eine Form, einen Sinn und einen Ursprung besitzt, dann muss man seine zeitliche Dimension mit in die Darstellung einbeziehen, man muss die Bewegungen berücksichtigen, die sich in diesem Raum vollziehen, rasant oder mit unerbittlicher Langsamkeit, alle Elemente, die dieser Raum aufgrund seiner gegenwärtigen und vergangenen Beziehungen enthält . ${ }^{1}$
}

1 Calvino, Italo: Savona: storia e cultura. In Ders.: Saggi 1945-1985. A cura di Mario Barenghi. 2 Bde. Mailand: Mondadori 1995, p. 2390. Übersetzt nach Zibaldone - Zeitschrift für italienische Kultur der Gegenwart (Tübingen) 32 Schwerpunkt Ligurien (2001), S. 6. 


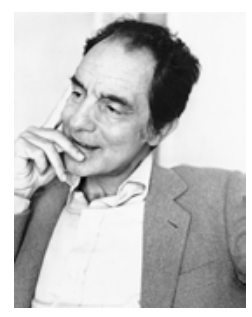

Abb. 16: Italo Calvino (Santiago de las Vegas, 1923 - Siena, 1985).

Der italienische Schriftsteller und Literaturtheoretiker trifft mit seiner Analyse von Räumen und deren Beschreibung präzise ins Schwarze. Und er tut dies mit einer geradezu traumwandlerischen Sicherheit, in welcher zu den drei Dimensionen des Raumes die vierte Dimension der Zeit hinzugenommen wird. Italo Calvino holt damit in die Beschreibung einer Räumlichkeit, einer Topographie, die Zeit als vierte Dimension mit herein, als eine Dimension, die im Grunde ständig jegliche Beobachtung im Raum begleitet, aber eine viel fundamentalere Rolle spielt bei der Beschreibung von Landschaften, Räumen oder Orten, als wir im allgemeinen glauben. Denn sie ist nicht nur auf der Ebene des Beobachtens selbst präsent, sondern im Grunde auf allen Ebenen von Wahrnehmung und Darstellung, von Empfindung und Beschreibung. Lassen Sie es mich an dieser Stelle der Vorlesung zumindest schon einmal erwähnen. Dabei will ich auch nicht unerwähnt lassen, dass ich Bewegungen hier nicht nur als motions, sondern auch als emotions verstehe. Aber dazu später mehr.

Bei diesen theoretischen Vorüberlegungen zur Vorlesung sei noch ein zweiter Gedanke hervorgehoben, der in der Folge wesentlich verfeinert und differenziert vorgetragen und exemplifiziert werden wird. Unsere Vorlesung behandelt Texte aus dem 15. Jahrhundert bis hin zu Beginn des 21. Jahrhunderts und erfasst damit einen Zeitraum - und dieser Ausdruck ist hier nicht umsonst wichtig -, den wir mit Blick auf die europäische Entwicklung, aber auch auf die europäische Expansion und die von ihr ausgehenden Bewegungen als den Zeitraum von Neuzeit, Moderne und Postmoderne bezeichnen können. Vielleicht ist es sinnvoll, an dieser Stelle eine kurze Exkursion zum Modernebegriff einzufügen, auf den wir noch mehrfach zurückkommen werden. Denn von unserem Modernebegriff hängt ab, welchen Sinn wir aus den Jahrhunderten seit der Frühen Neuzeit schlagen können und wie wir unsere eigene Zeit und deren Zukunft verstehen wollen.

Es dürfte wohl kaum übertrieben sein, wenn wir in der Bestimmung des Begriffs der Moderne die Gretchenfrage der zweiten Hälfte des 20. Jahrhunderts nicht nur in Europa, sondern gerade auch in den außereuropäischen Kulturen 
und Nationen erblicken. Leicht abgewandelt könnten wir formulieren: 'Sag' mir, was für Dich die Moderne ist, und ich sag' Dir, wer Du bist'.

Die Moderne wurde historisch sehr unterschiedlich definiert: Die einen sahen in ihr - wie etwa Humboldts Zeitgenosse Chateaubriand - das Zeitalter des von der heidnischen Antike abgesetzten Christentums; die anderen identifizierten sie mit dem Begriff der Neuzeit, der bekanntlich in andere europäische Sprachen als modern times oder les temps modernes übersetzt wird. Aus philosophischer Sicht wurde sie mit dem Projekt der Aufklärung oder Spätaufklärung und historisch mit der Zeit seit der Französischen Revolution identifiziert - zwei Bestimmungen, die von größtem Gewicht sind für eine geschichtlich reflektierte Bestimmung dessen, was wir unter 'der Moderne' verstehen dürfen.

Aber die Dinge sind auch hier komplexer und in mehr als einer Hinsicht komplizierter, verwirrender. Denn aus kulturtheoretischer oder literarästhetischer Perspektive wiederum schien nicht das letzte Drittel des Siècle des Lumières, sondern jenes des 19. Jahrhunderts mit dem Aufbruch in die Moderne gleichsetzbar zu sein. Modernismen jeglicher couleur - ob in der Literaturgeschichte oder in der Theologie, ob in der Kunstgeschichte oder speziell der Malerei - legen hiervon beredt Zeugnis ab. Andere Deutungsmuster folgten wiederum der Tradition, dass stets die Mitte eines Jahrhunderts die großen Umschwünge bringe; So sah man, um nur zwei Beispiele zu nennen, entweder nach den Revolutionen von 1848 oder aber auch nach dem Ende des Zweiten Weltkrieges die Zeit der Moderne gekommen. Damit nicht genug: Sprechen die einen (und es ist bisher die Mehrzahl) von der einen Moderne, so sprechen andere im Plural von den 'anderen Modernen', signalisieren die einen eine 'zweite Moderne' im Zentrum, so sehen die anderen eine 'periphere Moderne"2 an den sogenannten Rändern der Weltwirtschaft und des Kulturbetriebs. Fürwahr: Der Modernebegriff ist höchst verwirrend und gibt Raum für Spekulationen, die weniger die Position des Gegenstandes als jene der Betrachter beleuchten.

Doch legen wir uns - gewiss wohlreflektiert - in dieser Sache eindeutig fest. Denn ich möchte Ihnen nicht nur einen Fächer an Möglichkeiten aufzeigen und Sie dann mit dem Aufgezeigten alleine lassen, sondern auch klar eine

2 Die für die ungemein spannende, vorwiegend aus kulturtheoretischer Perspektive geführte lateinamerikanische Diskussion der neunziger Jahre prägenden Veröffentlichungen stammen von Sarlo, Beatriz: Una modernidad periférica: Buenos Aires 1920 y 1930. Buenos Aires: Ediciones Nueva Visión 1988; García Canclini, Néstor: Culturas híbridas. Estrategias para entrar y salir de la modernidad. México: Grijalbo 1989. Eine kritische Bewertung dieser Diskussion findet sich in Verf.: ¿Heterogeneidad cultural y homogeneidad teórica? Los 'nuevos teóricos culturales' y otros aportes recientes a los estudios sobre la cultura en América Latina. In: Notas (Frankfurt am Main) III, 7 (1996), S. 2-17. 
Position beziehen, die sie identifizieren und gewiss auch kritisch überdenken können und sollen. Ich möchte in unserer Vorlesung den Aufbruch in die Moderne und damit den Prozess der Moderne, der sich in der Folge freilich auffächern und auf verschiedene Modernen ausweiten sollte, auf jenen Zeitraum im letzten Drittel des 18. Jahrhunderts beziehen, in dem wir seit den Studien von Reinhart Koselleck und zuvor von Michel Foucault eine deutliche Verzeitlichungstendenz und die eigentliche Entstehung des Kollektivsingulars Geschichte erkennen können. Davor liegt die Frühe Neuzeit, die nicht zuletzt ganz wesentlich von der ersten Phase beschleunigter Globalisierung geprägt ist. Denn in der Tat sind es die Globalisierungsphasen, die ganz wesentlich in eine Zeitbestimmung einfließen, die scheinbar ganz allein auf Europa bezogen ist, tatsächlich aber ein Effekt von globalgeschichtlicher Bedeutung ist. Halten wir also fest: Die Frühe Neuzeit beginnt mit der ersten Phase beschleunigter Globalisierung, auch wenn sich viele Elemente ihrer späteren Entwicklung auch schon in ihrem Vorfeld aufzeigen ließen. Aber dies gilt auch für das Phänomen einer Beschleunigung dessen, was wir gemeinhin als Globalisierung bezeichnen.

Erst mit dem Aufstand der amerikanischen Kolonien Großbritanniens in der sogenannten Amerikanischen Revolution und sicherlich mehr noch in der Französischen Revolution von 1789 entwickelte sich definitiv ein Verständnis der Zeit, das nicht mehr vorrangig zirkulär geprägt, sondern unverkennbar offen, insbesondere ergebnisoffen, linear und zugleich vom Menschen beeinflussbar erschien. Wir sollten in der Aufzählung der Revolutionen gerade auch an dieser Stelle auf keinen Fall die Haitianische Revolution außer Acht lassen, denn war sie es doch, die just im gleichen Zeitraum nicht nur eine antikoloniale Revolution wie die Amerikanische in Gang setzte, sondern mehr noch die Revolution gegen die Sklaverei erfolgreich durchführte und damit weit über die Entstehung der USA hinausreichte, welche - dies ist evident - die Sklaverei sehr wohl beibehielten und bis in die Gegenwart eine Rassendiskriminierung kennen. Dass damit die tragische Geschichte Haitis begann, hat weniger mit dem Erfolg der Revolution gegen die Sklaverei zu tun als mit der Tatsache, dass sich der 1804 neu gegründete Staat Haiti allen möglichen Geldforderungen und Entschädigungen gegenübergestellt sah, die ihn im 19. Jahrhundert buchstäblich erdrosselten. Halten wir also fest, dass in denselben Zeitraum die Haitianische Revolution hineinfällt, welche ebenfalls eine ergebnisoffene Verzeitlichungsstruktur prägt und von Menschen getragen wurde, welche ihr Schicksal selbst bestimmen wollten.

Wenn wir die Haitianische Revolution berücksichtigen und in unseren Modernebegriff miteinbeziehen, dann weichen wir darin erheblich von einem bisherigen gängigen Geschichtsmodell $\mathrm{ab}$, in dem diese Revolution bislang in den Revolutionstheorien, aber auch innerhalb des Moderne-Denkens keine oder keine wirkliche Rolle spielen durfte. Wenn wir von einer europäischen Doppelrevolution 
sprechen können und darunter sowohl die Französische (und damit politische) Revolution und zugleich die Industrielle Revolution (in England) verstehen, so müssen wir lernen, auch eine Amerikanische Doppelrevolution zu denken, bei der es nicht nur um die US-amerikanische Revolution gehen kann, sondern die Haitianische Revolution unbedingt mitgedacht werden muss. Kurzum: Die Haitianische Revolution ist das Phänomen einer europäischen Moderne, die sich fernab Europas zu anderen Modernen hin öffnet. Moderne muss also global gedacht werden nicht im Sinne von Peripherie und Zentrum, sondern von sich miteinander verknüpfenden Modernen. Es ist an der Zeit, die Vielgestaltigkeit der Modernen weltweit zu denken.

Die Moderne wie die Modernen werden folglich - und dies ist der entscheidende Punkt - durch ein grundlegend verändertes Verhältnis zur Zeit bestimmt. Damit setzte zugleich jene Verzeitlichung in den unterschiedlichsten Bereichen ein, die unser Leben bis heute ganz selbstverständlich betrifft, obwohl die Zeit natürlich keine Erfindung des ausgehenden 18. Jahrhunderts war. Aber in diesem Zeitraum bildete sich eine Konzeption von Geschichte heraus, wie sie bis weit ins 20 . Jahrhundert hinein dominieren sollte und auch heute noch immer präsent ist.

Verzeitlichungsstrukturen beherrschen die Moderne wie die Modernen. Die Verzeitlichung aller Lebens- und Wissensbereiche ließe sich an einer Vielzahl von Beispielen aufführen, die wir miteinander durchbuchstabieren könnten. Sie wurde von Michel Foucault etwa daran aufgezeigt, dass seit dem letzten Drittel des 18. Jahrhunderts und mit jeweils eigenen zeitlichen Entwicklungen in den einzelnen sich herausbildenden Disziplinen Vorstellungen zum Tragen kamen, die die Zeit als wesentliche Kategorie mitzudenken begannen und den Objekten, den Gegenständen der Forschung, jeweils ihre eigene Zeitlichkeit einschrieben. Die Phänomene standen nun nicht länger nebeneinander in einem einzigen Tableau der Naturgeschichte - hierüber hat Wolf Lepenies ${ }^{3}$ das Entscheidende gesagt -, sondern zwischen ihnen bildete sich zunehmend eine zeitliche Verkettung im Sinne einer Entwicklung heraus, die dann mit Darwin in Richtung einer eigentlichen Evolutionstheorie radikalisiert werden sollte, aber keineswegs etwas radikal Neues an sich darstellte. Denn die Verzeitlichung aller Wissensbestände war längst ein Faktum innerhalb der Wissenschaftsgeschichte geworden, von dem aus sich das Wissen neu anordnen ließ - und zwar in allen Disziplinen. Die verschiedenen Fossilien etwa ordneten sich nicht mehr im Raum als voneinander

3 Vgl. hierzu Lepenies, Wolf: Das Ende der Naturgeschichte. Wandel kultureller Selbstverständlichkeiten in den Wissenschaften des 18. und 19. Jahrhunderts. Frankfurt am Main: Suhrkamp 1978. 
geschiedene Artefakte an, die ihre jeweilige Position innerhalb eines Tableaus einnahmen, sondern wurden in eine historische Abfolge gestellt, die ihre Veränderungen und Evolutionen in den Fokus der Betrachtung rückte. Episteme und Epistemologie hatten sich grundlegend verändert: Alles wurde von einer allgegenwärtigen Zeitvorstellung durchdrungen.

Wir werden noch viele weitere Charakteristika von Moderne kennenlernen, doch sei an dieser Stelle bereits festgehalten, dass der Einzug von Verzeitlichungsstrukturen grundlegend für die Moderne ist - und ich meine damit auch die Verschiedenheit der Modernen. Daher lässt sich die Moderne vor allem als ein von der zeitlichen Dimension, also der vierten Dimension, beherrschter Zeit-Raum begreifen und somit als eine Epoche, in welcher alles unter den Gesichtspunkt von Zeit und Verzeitlichung gerät.

Was sich seit Ende der sechziger Jahre in Teilen Europas, der USA und zum Teil schon früher - in Lateinamerika herausbildete, und was wir später als Postmoderne bezeichnen hörten (und bezeichnen würden), impliziert eine gewisse Abkehr von dieser beherrschenden Verzeitlichungsstruktur, in der die Geschichte zur Grundlage jeglichen Wissens und jeglicher Erfahrung geworden ist, ja zu einer Kraft avancierte, deren Präsenz so selbstverständlich wurde, dass sie eigentlich keiner gesonderten Erwähnung bedurfte. Die Postmoderne ihrerseits verweist in wesentlich stärkerem Maße auf Strukturen des Raumes: Man könnte analog zur Verzeitlichung auch von einer Verräumlichung sprechen, die sich in ihren neuen Denkstrukturen deutlich ausdrückt. Doch schauen wir uns das etwas näher an.

Insistiert die Postmoderne also innerhalb eines gemeinsamen Raumes von Moderne und Postmoderne wesentlich prononcierter auf den Raumstrukturen und damit auf dem spatialen Element - den ersten drei Dimensionen, auf die wir gleich noch einmal wesentlich ausführlicher zurückkommen werden -, so zeigt sich zugleich, dass sich möglicherweise in der Gegenwart etwas entwickelt, was im Grunde wesentlich stärker diese Dimensionen aufeinander bezieht und nicht einseitig die Dimension der Zeit und damit die Verzeitlichung oder die Dimensionen des Raumes und damit der Verräumlichung, sondern ein Zusammenspiel zwischen diesen Dimensionen und damit die Bewegung privilegiert und betont. Genau hier siedelt sich mein Argument an: Wir haben es mit einer Privilegierung des Bewegungselementes, also der Bewegung an sich, zu tun. Dies bedeutet nicht, dass Bewegung nun etwas ganz Neues wäre: Wir finden sie in allen Zeiten und Epochen. Aber es bedeutet, dass ihr eine privilegierte Aufmerksamkeit zuteilwurde und ihr Gewicht gar nicht überschätzt werden kann.

Es könnte also sein, dass wir gerade heute - genauer gesagt: seit etwa vier Jahrzehnten - jene Veränderungen erleben, in der wir durchaus eine globale Tendenz erkennen dürfen, ohne freilich diese Vorstellungen einfach auf unseren 
Planeten zu stülpen und unbesehen zu generalisieren. Die Metaphorik des Netzwerks ist dabei derzeit in aller Munde: vom Netzwerk der miteinander vernetzten Computer oder dem Weltweiten Gewebe des Internets bis hin zu weltweiten Netzwerken in der Ökologiebewegung oder auch - ganz anders wieder - in bestimmten religiösen, ideologisch motivierten oder auch terrroristischen Netzwerken, von denen ständig in unseren Nachrichten die Rede ist. Das Jetztzeitalter ist das Netzzeitalter.

Ich kann hier nicht ausführlich auf die Verschiedenartigkeit von NetzVorstellungen eingehen. Aber klar ist, dass wir zentrierte Netzstrukturen haben und nicht-zentrierte davon unterscheiden können; dass es starre Netze gibt, die nicht verändert werden dürfen, aber auch hochdynamische, bei denen sich ständig irgendwelche Verbindungen zwischen einzelnen Knotenpunkten verändern und neue Querverbindungen bilden. Faszinierend ist, wie sehr sich Netzwerkstrukturen je nach den entsprechenden Leitvorstellungen voneinander unterscheiden können. Dabei würde ich am Ende lebendige Netzwerkstrukturen privilegieren: also solche, die sich eigenständig in permanenter Veränderung befinden. Ja: Es ist Leben im Netz - oder es kann zumindest Leben im Netz sein. Doch dies ist nur eine Unterart von Netzwerkstrukturen, mit denen wir uns auch und gerade in der Reiseliteratur beschäftigen müssen.

In jenen Überlegungen, die ich hier zum Auftakt der Vorlesung nur kurz anreißen kann, die ich aber noch wesentlich ausführlicher diskutieren werde, spielen nicht statische Netzwerke, sondern (lebendige) Bewegungen, wenn Sie so wollen bewegliche Netzwerke oder Netzwerke in Bewegung, eine fürwahr zentrale Rolle. Denn die planetarische Erfassung durch weltumspannende Netzwerke hat keineswegs - wie dies die Postmoderne dominant entwickelte - eine Verräumlichung des Denkens vorbereitet und durchgesetzt, sondern die Möglichkeit geschaffen, die zeitliche Dimension im Sinne einer Bewegung ganz im Sinne Italo Calvinos miteinzubeziehen. Dimensionen des Dynamischen haben sich hier eröffnet und stoßen weit die Türen unseres Verständnisses auf. Wir haben es mit Bewegungselementen, mit Vektoren, auf Schritt und Tritt zu tun. Man könnte dies als Vektorisierung bezeichnen und entsprechend begrifflich implementieren - aber wir wollen nicht gleich zu Beginn dieser Vorlesung zu viele abstrakte Begriffe wählen. Vektorisierung ist in jedem Falle ein Begriff, der nicht nur auf eine gegenwärtige Dynamik abzielt, sondern die historisch akkumulierten Bewegungen immer schon in sich gespeichert bereit hält und zugleich auch künftige Bewegungen, die Bewegungen des Künftigen, in sich in der Jetztzeit aufgenommen hat. Vektorisierung ist folglich ein Schlüssel, um unsere Epoche zu verstehen.

Vor diesem Hintergrund beginnen wir zu begreifen, warum nun Reisen und Reisebewegungen und deren Untersuchung - die lange Zeit ganz und gar nicht 
zum Teil des literaturwissenschaftlichen Kanons zählten und im Grunde ein freudloses Randdasein fristeten - heute eine so große und entscheidende Rolle zukommt und warum es sich lohnt, sich ausführlich mit der Entwicklung und den Veränderungen innerhalb der Reiseliteratur zu beschäftigen. Dank der Reiseliteratur und ihren Vektorisierungen können wir uns in die Lage versetzen, unsere Epoche in einem gänzlich neuen und anderen Licht erscheinen zu lassen. Wir können alles im Zeichen der Bewegung, der Speicherung von Bewegungen aller Art, begreifen. Das wollen wir in der Folge auch mit großer Freude tun.

Doch noch einmal zurück zu unserem italienischen Ausgangspunkt. Wir haben uns zu Beginn also mit Italo Calvino die Frage gestellt, wie man einen Ort oder einen bestimmten Raum überhaupt beschreiben kann. Wir können und sollten nun aber eine zweite Frage stellen, die ebenfalls auf den ersten Blick banal erscheinen könnte: Wie lässt sich eine Reise schreiben und beschreiben?

Bevor wir auf die verschiedenen Möglichkeiten eingehen, möchte ich an dieser Stelle zunächst aus einer anderen Perspektive die Frage erörtern, wie man eigentlich ein (bereistes) Land beschreiben kann. Welche Mittel stehen uns hierfür zur Verfügung?

An dieser Stelle knüpfen wir an unsere Überlegungen zu Karten, Weltkarten und Kartenwerken an. Seit unvordenklichen Zeiten werden Länder und Regionen mit Hilfe von Karten beschrieben, mithin kartographiert. Die frühesten Karten gehen meines Wissens bis in die Jungsteinzeit zurück. Frühe Formen des Schreibens von Karten sind hier folglich ebenso zu berücksichtigen wie die modernsten, die wir mit Hilfe von Google Maps erstellen. Hier geht es also um die Verwandlung in verschiedene Formen von Schrift, graphein - und dies darf durchaus auch die Lichtschrift, die Photographie miteinschließen. Denn nicht selten haben wir es bei den Reiseberichten ganz wesentlich mit inter- und mit transmedialen Zeugnissen und Erzeugnissen zu tun, also mit Formen, in denen sich unterschiedliche Medien begegnen und queren.

Von jeher stellten sich die Geographen die Frage nach dem Maßstab und der Unterlage dieser Kartenschrift, die ihrerseits in engster Beziehung zu Philosophie und Literatur stehen und keineswegs nur banale Voraussetzungen jeglicher Kartenanlage darstellen. Hören wir uns dies in einer von mir übersetzten Passage eines Essays aus den Otras inquisiciones des großen argentinischen Schriftstellers und Erfinders philosophischer Fiktionen Jorge Luis Borges an:

Die Erfindungen der Philosophie sind nicht weniger phantastisch als jene der Kunst: Josiah Royce formulierte im ersten Band des Werkes The World and the Individual (1899) die folgende: „Stellen wir uns vor, ein Teil der Erdoberfläche Englands wäre vollkommen eingeebnet worden, und darauf würde ein Kartograph eine Karte Englands zeichnen. Das Werk ist vollkommen: Nicht ein einziges Detail der Erdoberfläche Englands, so winzig es 
auch wäre, würde auf der Karte fehlen; alles findet hier seine Entsprechung. In diesem Falle muss diese Karte eine Karte der Karte der Karte und so weiter bis ins Unendliche enthalten.“

Warum beunruhigt es uns, dass die Karte in der Karte enthalten ist und tausend und eine Nacht im Buch Tausend und eine Nacht? Warum beunruhigt es uns, dass Don Quijote ein Leser des Quijote ist und Hamlet ein Zuschauer von Hamlet? Ich glaube, den Grund gefunden zu haben: Derartige Inversionen legen uns nahe, dass wir, wenn schon die Figuren einer Fiktion Leser oder Zuschauer sein können, als Leser und Zuschauer selbst fiktiv sein könnten. Im Jahre 1833 bemerkte Carlyle, dass die Weltgeschichte ein unendliches heiliges Buch sei, das alle Menschen schreiben und lesen und zu verstehen suchen, und in welchem sie auch sich selber schreiben. ${ }^{4}$

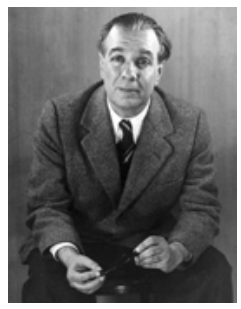

Abb. 17: Jorge Luis Borges (Buenos Aires, 1899 - Genf, 1986).

In dieser Passage begegnet uns das für Jorge Luis Borges' Schreiben typische Element der Rationalität und der Verunsicherung, der geistigen Durchdringung und jenes Unheimlichen, von dem uns Sigmund Freud die heimliche Unheimeligkeit zeigte. In diesem Schlussteil eines Textes aus Jorge Luis Borges' Otras inquisiciones wird nicht nur die phantastische Erfindungskraft der Philosophie mit jener der Kunst auf eine Stufe gestellt und damit zugleich die Grenze in Frage gestellt, welche die fiktionalen von den diktionalen' ${ }^{5}$ (oder 'expositorischen' beziehungsweise 'nicht-fiktionalen') Texten trennt. Der argentinische Autor geht in dieser Passage weit über die häufig von ihm gestaltete Lieblingsidee $^{6}$ hinaus, indem er sich auf ein aus seinem Geburtsjahr stammendes apokryphes Werk stützt und mit ihm gerade jene Kunst verknüpft, die doch als die im engsten Sinne wirklichkeitsbezogenste gelten darf, die Kartographie. Shakespeare und Cervantes müssen tatenlos zusehen, wie ihre großartigen Fiktionen mit einer Karte von England verglichen werden, die wir als ein eingeebnetes

4 Borges, Jorge: Magias parciales del Quijote. In Ders.: Prosa completa. 4 Bde. Barcelona: Bruguera 1980, Bd. 2, S. 174.

5 Vgl. hierzu Genette, Gérard: Fiktion und Diktion. Aus dem Französischen von Heinz Jatho. München: Fink 1992.

6 Vgl. hierzu auch Ette, Literatur in Bewegung, S. 247-268. 
Land begreifen dürfen. Aber was verbindet die Kartographie eines Landes mit dem Schreiben eines Landes? Wie lassen sich die Reisebewegungen des Don Quijote in der spanischen Mancha mit dem Schreiben einer platten Karte von England vergleichen?

Die Kartographie 'klebt' gleichsam an der Erdoberfläche, wandelt sie zugleich aber in Zweidimensionalität um. Die dritte Dimension wird in diese Zweidimensionalität durch bestimmte konventionelle Zeichen, die sich über die Zeit wandeln können, sorgfältig eingetragen. Ein mimetisches Streben nach Vollständigkeit ist der Kartographie eigen, seit Menschen Umrisse von Territorien auf den Erdboden zeichneten oder in das Gestein einritzten. Eine topographische oder geographische Karte gibt uns das Bild unserer Welt möglichst präzise wieder, so dass wir sie unmittelbar benützen können, um uns in der Wirklichkeit, in unserer komplexen Welt, auf vereinfachte Weise zurechtzufinden. Es bedurfte nicht des vor einigen Jahren erschienenen Atlas der Erlebniswelten, ${ }^{7}$ um uns vor Augen zu führen, dass ein kartographisches Abbild der Welt in erster Linie ein Bild der Welt und somit das Fiktionale gerade im vertrauten Kartenbild gut aufgehoben ist. Finden und Erfinden liegen nicht nur etymologisch nahe beieinander ${ }^{8}-$ und wir haben ihr Zusammenwirken im Leben und Erleben bereits eingehend in den Kartenwelten untersucht. Wir können Finden und Erfinden anders miteinander verknüpfen, insofern wir beide vom Leben her in einem anderen Licht erscheinen lassen können: in dem des eigenen oder fremden Erlebens.

Folglich fügte Alexander von Humboldt (vgl. Abb. 72: Porträt Alexander von Humboldts) seinem Atlas géographique et physique du Nouveau Continent eine Abfolge kartographischer Darstellungen bei, die ausgehend von einer Karte des Jodocus Hondius aus dem Jahre 1599 die Geschichte der geographischen Darstellungen von El Dorado bis in seine unmittelbare Gegenwart - die letzte Karte von Surville stammte aus dem Jahre 1778 - nachzeichnete. ${ }^{9}$ Dabei war sich der preuBische Reiseschriftsteller und Gelehrte durchaus des fiktionalen Charakters derartiger Karten bewusst: ja, er führte gerade die Fiktionalität dieser Karten vor Augen.

7 Vgl. Klare, Jean / Swaaij, Louise von: Atlas der Erlebniswelten. Mit Texten von Ilja Maso und Saskia Sombeek. Frankfurt am Main: Eichborn Verlag 2000.

8 Zur 'Eigenart der geographischen Hoffnung' vgl. Bloch, Ernst: Das Prinzip Hoffnung. Bd. 2. Frankfurt am Main: Suhrkamp 1973, S. 874-880.

9 Ein Abdruck dieser Kartenfolge des Atlas géographique (Tafel 14) findet sich leicht zugänglich in Ette, Ottmar (Hg.) / Humboldt, Alexander von: Reise in die Äquinoktial-Gegenden des Neuen Kontinents. Mit Anmerkungen zum Text, einem Nachwort und zahlreichen zeitgenössischen Abbildungen sowie einem farbigen Bildteil. Bd. 1-2. Frankfurt a. M.. Insel 1991, Bd. 2, S. 1343-1345. 
Dabei machte er auf den erfindungsreichen Charakter der von ihm signalisierten Karten aufmerksam. Es ist eine Eigenart dieser 'geographischen Hoffnungen', Einzelheiten aufzuhäufen und sie mit Autorität(en) zu verknüpfen. Die von europäischen Kartographen angefertigten Darstellungen des Mythos vom Vergoldeten König, den Europäer unterschiedlicher Breitengrade unter tatkräftiger Mithilfe indianischer Informanten weitab ins südamerikanische Binnenland projizierten, führten nicht nur jahrhundertelang Conquistadoren, Abenteurer oder Forschungsreisende in Gebiete, in denen keine goldenen Schätze zu heben waren, sondern machten auch vor dem Namen Alexander von Humboldts nicht Halt. Daher musste der Zerstörer geographischer Mythen in seinem Reisebericht erstaunt feststellen, dass die historische Aufarbeitung des Mythos nicht zu dessen Auflösung geführt hatte, sondern auf den Mythenforscher selbst übergesprungen war:

Diese Notizen hatte der Geschichtsschreiber der Grenzexpedition an Ort und Stelle gesammelt, und er hätte wohl nicht geglaubt, daß La Cruz und Surville richtige Darstellungen und alte Vorstellungen vermengen und auf ihren Karten das Mar Dorado oder Mar Blanco wieder zum Vorschein bringen würden. So kommt es, daß, obgleich ich seit meiner Rückkehr aus Amerika vielfach den Beweis geführt, daß ein Binnenmeer, aus dem der Orinoco entspränge, gar nicht existiert, in neuester Zeit unter meinem Namen eine Karte erschienen ist, auf der die Laguna de Parima wiederum auftritt. ${ }^{10}$

Der Schöpfer des Kosmos sah sich hier in seine eigene Geschichte der Mythenzerstörung verwickelt. Seine Beschäftigung mit Mythen führte dazu, dass sich der Mythos nun auch mit ihm beschäftigte und auf sein eigenes Werk übergriff. Alexander von Humboldt, dessen Wissenschaft in der Tat ein neues Weltbild schuf, das sich in auf den ersten Blick so entlegenen Gebieten wie der Medizingeographie auch im Kartenbild niederschlug, ${ }^{11}$ hatte die Existenz des Mythos durch die Wiedergabe vorgängiger, 'erfundener' kartographischer Darstellungen wider Willen nur auf eine neue Stufe gehoben. Mit Hilfe von Karten sind Mythen nicht auszurotten: Der Mythos, die Fiktion, schreibt sich unverzüglich in sie ein. Wir begreifen, in welchem Maße neue Karten eine lange Abfolge alter Karten, wie sehr neue kartographische Weltbilder alte Bilderwelten in sich aufnehmen

10 Ebda., S. 1349. Die Karte war 1818 in Wien erschienen und berief sich im Titel auf Humboldts längst nicht mehr nur in Wissenschaftskreisen renommierten Namen.

11 Vgl. die aufschluss- und kartenreiche Studie von Rupke, Nicolaas A. / Wonders, Karen E.: Humboldtian Representations in Medical Geography. In: Rupke, Nicolaas A. (Hg.): Medical Geography in Historical Perspective. ('Medical History', Supplement No. 20). London: The Wellcome Trust Centre for the History of Medicine at UCL 2000, S. 163-175. 


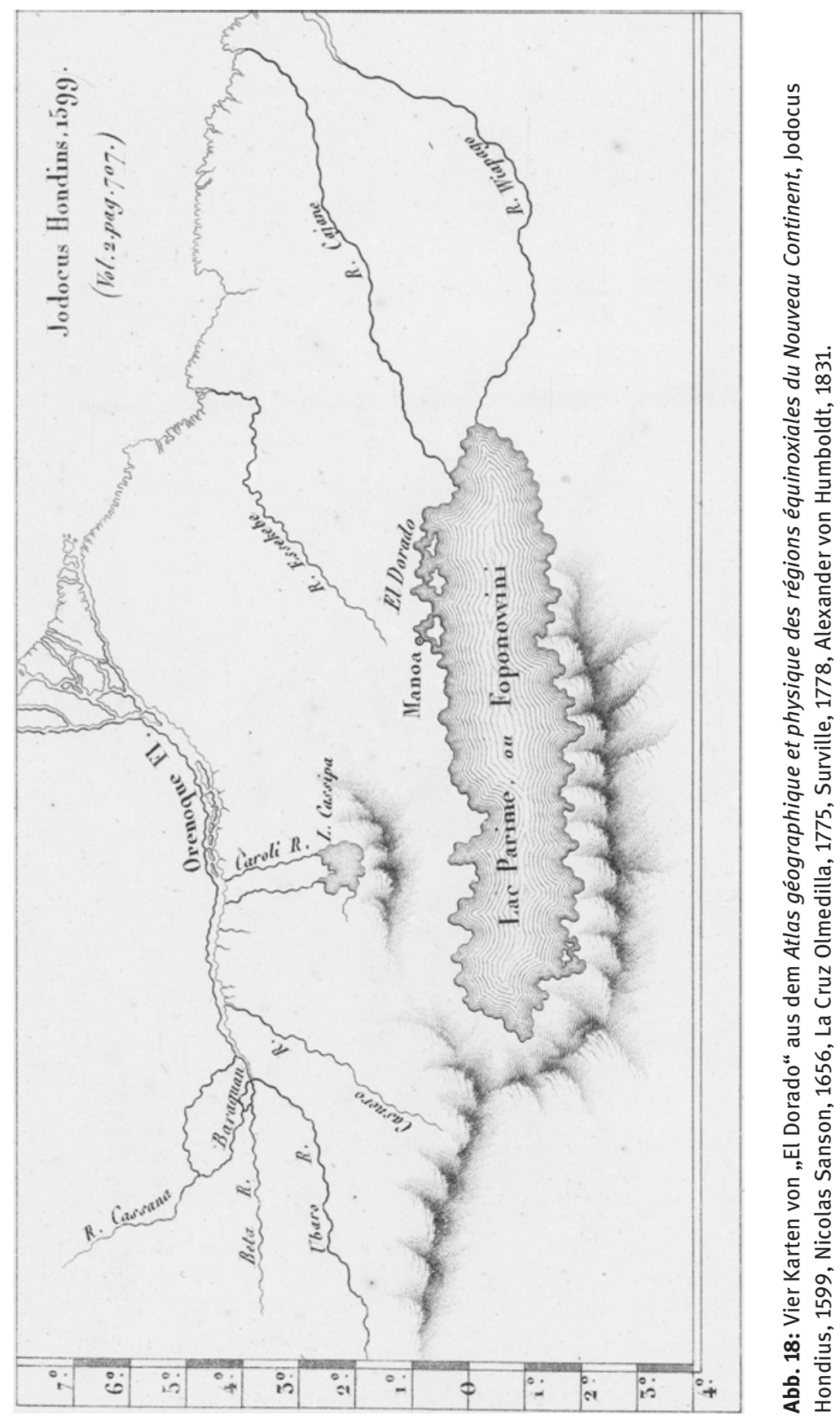




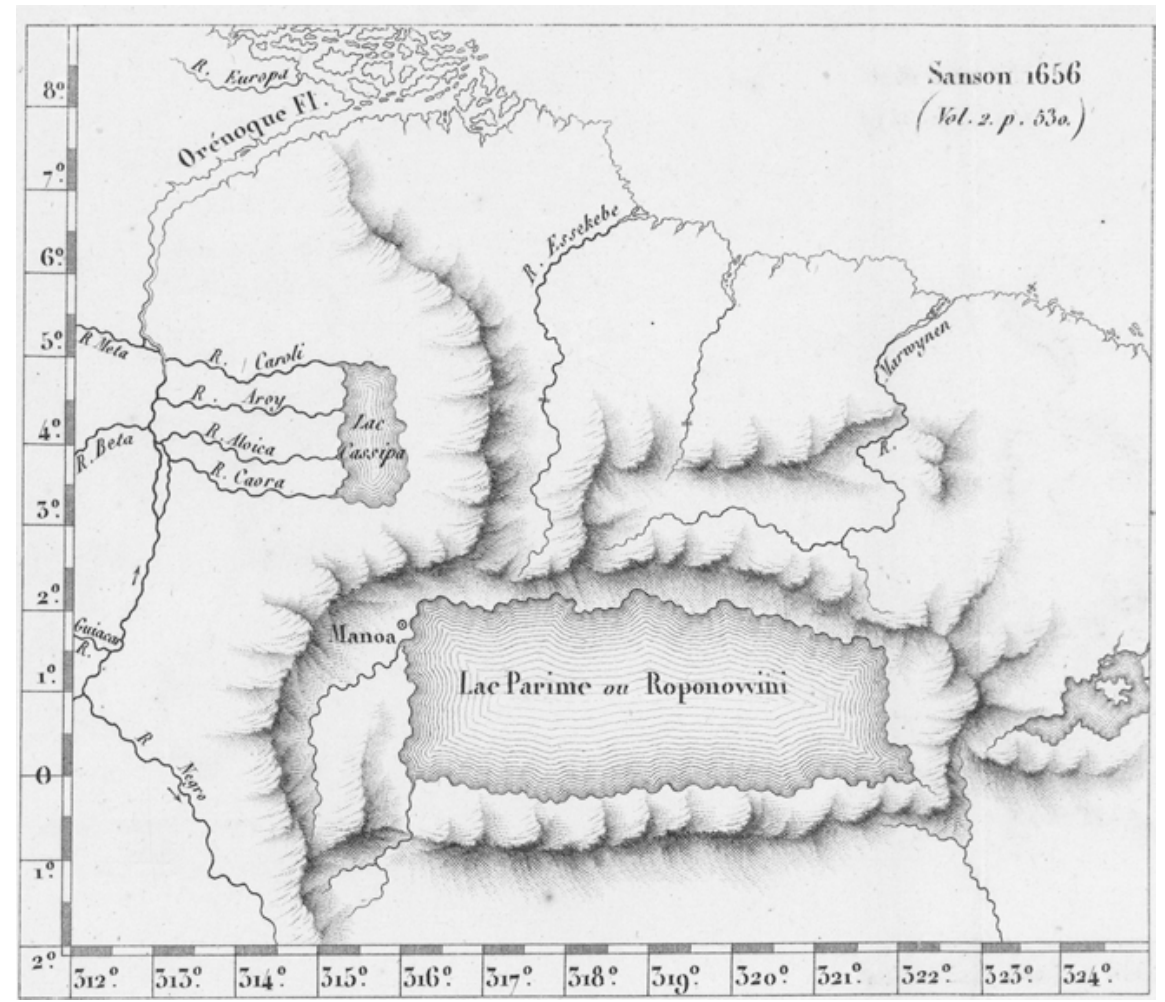

Abb. 18 (fortgesetzt)

und weitertransportieren. Und dies ganz wie in den Literaturen der Welt auch in den Karten der Welt: ad infinitum.

Doch kehren wir zum hintersinnigen Text von Jorge Luis Borges und seinem Rückgriff auf die Überlegungen eines (erfundenen) Anderen zurück. Er verdeutlicht, dass eine noch so präzise ausgeführte Karte Englands stets eine weitere Karte, ein weiteres Kartenbild in sich tragen muss, das um der Genauigkeit willen wiederum eine weitere Karte enthält, so dass sich auch hier ad infinitum kartographische Darstellungen ineinander schachteln, ein Verfahren, das Kartenschreiben mit Schreiben teilt und im Bereich der Literaturwissenschaft in Rückgriff auf André Gide als mise en abyme, als Spiegelung der Gesamtheit in einem einzigen modellhaften Punkt, bezeichnet wird. Wir haben es hier im vollen Wortsinne mit einem Fraktal zu tun. Um es mit Gertrude Stein zu sagen: A rose is a rose is a rose oder: 'Eine Karte ist eine Karte ist eine Karte.' 


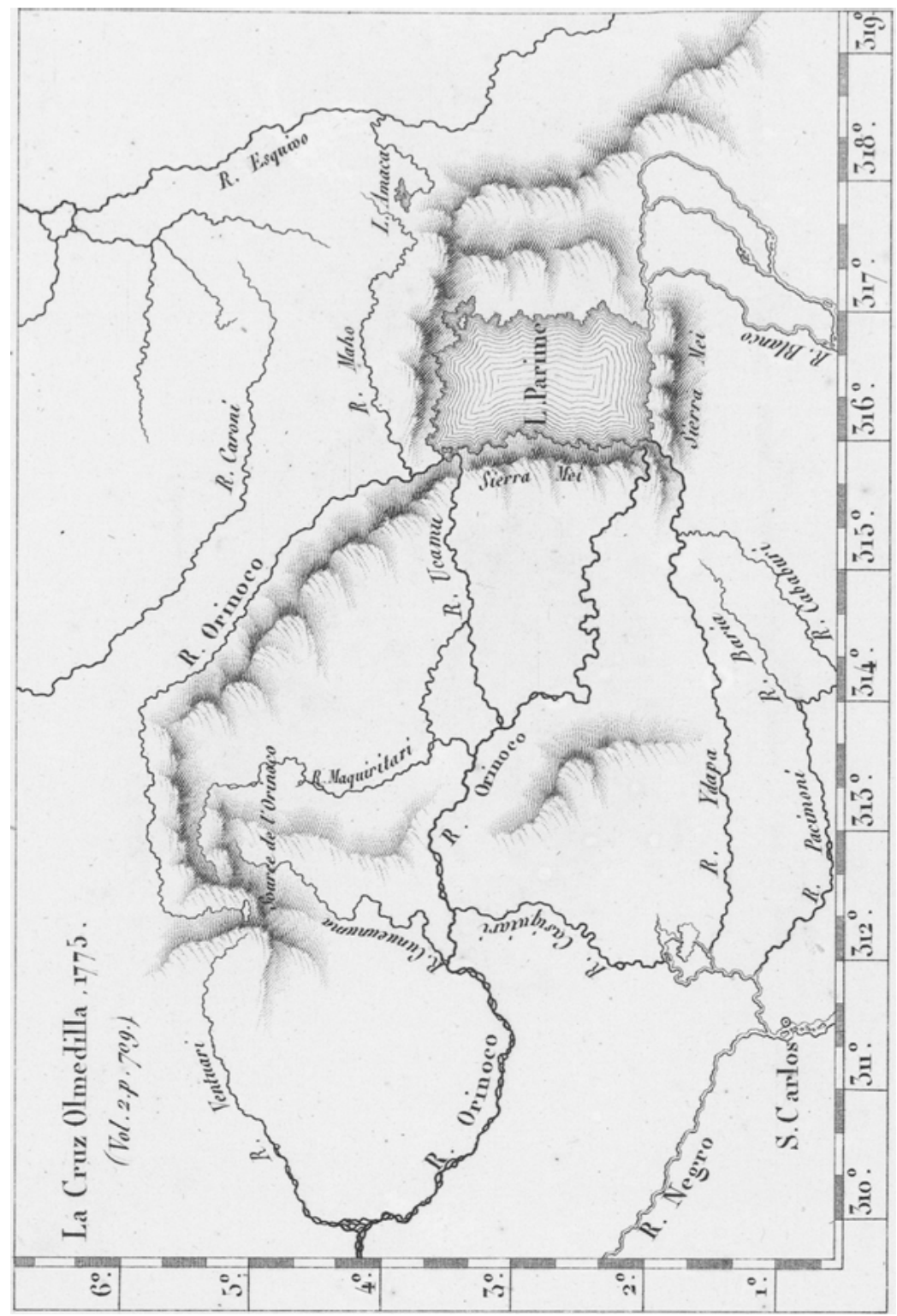

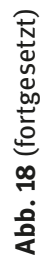




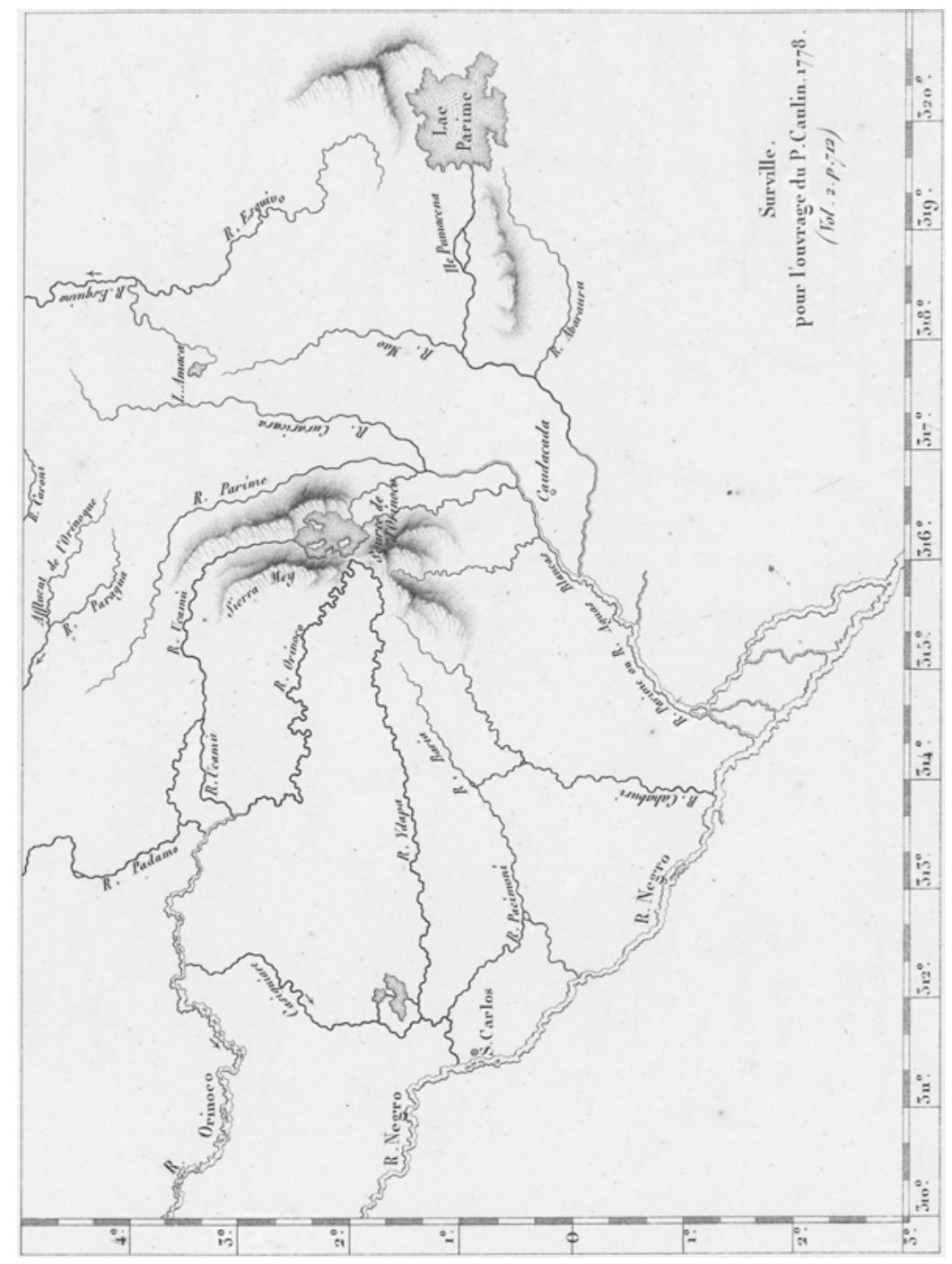

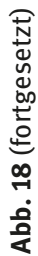


Doch der argentinische Schriftsteller ging noch weiter, denn die wechselseitige Selbstbezüglichkeit und Selbstähnlichkeit blieb nicht bei den kartographischen Darstellungen stehen. Ihre Mimesis - die in ihrer Tradition seit der Antike stets Nachahmung und Darstellung ist und sich dabei ebenso auf die Natur wie auf von Menschenhand geschaffene Vorlagen und Modelle bezieht durchkreuzt die Grenze zwischen Kunst- beziehungsweise Kartenwerk und Betrachter und springt auf Autor wie Lesepublikum über. Wie im zweiten Teil von Cervantes' Don Quijote, wo der Protagonist zum Leser des ersten Teiles seiner eigenen (Lese-) Abenteuer wird, ergreift auch die Weltgeschichte - in Borges' Entwurf von Carlyle - ihre Urheber und ihre Betrachter und verwandelt sie zugleich in Subjekte und Objekte dieser selben Weltgeschichte, dieser selben Weltkarte.

Im Bild der Weltgeschichte wie im Kartenbild eines Landes ist dessen $\mathrm{Au}$ torfigur stets in vielfachen Spiegelungen enthalten. Ein kleines Beispiel, um diese Aussage zu veranschaulichen. Das augenfälligste Beispiel ist die Tatsache, dass Humboldt in eine detaillierte Karte des Flussnetzes auch Hinweise auf eigene, in den Amerikanischen Reisetagebüchern und im Reisebericht festgehaltene Anekdoten einfügte. Allzu leicht vergessen wir, dass der objektive, aus großer Höhe geworfene und gleichsam absolute Blick, wie er in einem Kartenbild festgehalten ist, ein komplexes Geflecht aus Messungen, Beobachtungen, Konventionen und Erfahrungen ist und keineswegs nur dokumentiert. Alexander von Humboldts Orinoco-Karte ist ohne seine Reise nicht vorstellbar, und doch hat diese Reise nur einen kleinen Teil des in ihr dargestellten Gebietes wirklich in der Fläche erkundet. Was aber ist mit jenen riesigen Teilen, welche Humboldt überhaupt nicht besucht und selbständig eingezeichnet hat? Wo liegen die Quellen für diese Darstellung weiter Kartenbereiche? Karten wie Weltgeschichten können es also in sich haben: Sie enthalten ihren Verfasser gleichsam (und noch einmal) ad infinitum.

Doch es gibt noch einen zweiten Text von Jorge Luis Borges - der oftmals mit guten Gründen als einer der 'Väter der Postmoderne' bezeichnet wurde, da er uns vor allem grundlegende Einsichten in die Moderne und deren unterschiedliche Ausprägungen vermittelte -, in dem wir wichtige Hinweise für unsere Deutung Alexander von Humboldts finden können. Genaugenommen stammt dieser Text nicht von Borges selbst, sondern von Bustos Domecq, jener Autorfigur, die der Argentinier gemeinsam mit seinem Landsmann und Freund Adolfo Bioy Casares geschaffen hatte. Auch Bustos Domecq bedient sich in seinen Crónicas desselben parasitären Schreibverfahrens, indem er auf ein 'vergessenes' Werk des 17. Jahrhunderts, die Viajes de Varones Prudentes ('Reisen vorsichtiger Männer') zurückgreift, wo von einem weit entfernten Reich berichtet wird, in welchem die Kunst der Kartographie bis zur 
Vollendung entwickelt worden sei. ${ }^{12}$ Immer also ist es die Kartographie, die uns bei der Beschäftigung mit den unterschiedlichsten Formen der Reiseliteratur über den Weg läuft oder in die Quere kommt, ganz wie Sie wollen. Dort habe die „Karte einer einzigen Provinz die Größe einer ganzen Stadt“ eingenommen und „die Karte des Reiches gar eine ganze Provinz““. ${ }^{13}$ Im Ringen um eine noch höhere Präzision und Vollständigkeit seien die Colegios de Cartógrafos dann aber noch einen entscheidenden Schritt weiter gegangen:

Im Laufe der Zeit genügten diese maßlosen Karten nicht mehr den Ansprüchen, und die Kartographenvereinigungen erstellten eine Karte des Reiches, welche die Größe des Reiches einnahm und aufs Genaueste mit ihm übereinstimmte. ${ }^{14}$

Eine Karte des Reiches in der Größe des Reiches? Nachfolgende Generationen aber hätten den Sinn derartiger Kartenwerke nicht mehr verstanden und diese Karte „gnadenlos den Fährnissen der Sonne und der Winter ausgeliefert““. ${ }^{15}$ Aus jener Zeit seien einige Überreste dieser Karte auf uns gekommen, so dass man noch heute „in der Wüste des Westens“ die „Ruinen der Karte“ finde, „die von Tieren und Bettlern bewohnt werden“ - und „im ganzen Land“, so der bedeutungsvolle Schluss dieses Auszugs, gebe es keine ,andere Reliquie der geographischen Disziplinen“. 16

Sind vom geographischen Streben nach Abbildung der Welt nur noch Ruinen übrig geblieben? Gibt es hier nicht eine Tendenz, die jegliche Repräsentation von Welt schlicht ad absurdum führt?

Ähnlich wie in der Karte Englands hat sich aus Gründen der gewünschten Präzision ein Maßstab 1:1 ergeben. Doch wird in diesem Falle keine interne Verschachtelungsstruktur, sondern vielmehr eine Verwandlung des künstlerischen Artefakts der mimetisch-kartographischen Wiedergabe der Welt in die Wirklichkeit der Welt herausgearbeitet. Schließlich haben wir es mit einer Karte zu tun, die bewohnt wird, die also der Erde gleich ist und ihrerseits ein Territorium darstellt, in dem sich wohnen und leben lässt. Mit anderen Worten: Die Kartenschrift spiegelt das Reich nicht ab, sie ist ein Teil des Reiches selbst. Und doch ist die Karte des Reiches noch immer ein Modell, wurde von Menschen, wurde

12 Borges, Jorge Luis / Bioy Casares, Adolfo: Naturalismo al día. In: Borges, Jorge Luis: Obras Completas en colaboración. Barcelona: Emecé Editores ${ }^{4}$ 1997, S. 316; vgl. hierzu Scheines, Graciela: Las parodias de Jorge Luis Borges y Adolfo Bioy Casares. In: Cuadernos Hispanoamericanos (Madrid) 505-507 (Juli - September 1992), S. 532f.

13 Borges / Bioy Casares, Naturalismo al día, S. 316.

14 Ebda.

15 Ebda.

16 Ebda. 
von Kartographen eigens dafür hergestellt, sich der Wirklichkeit mit einem Modell im Maßstab 1:1 anzunähern.

Halten wir noch einmal deutlich fest: Die Mimesis vermischt sich unauflöslich mit dem Gegenstand ihrer Darstellung und verwandelt sich in die Wirklichkeit selbst, die bewohnbar und heimisch wird, aber auch verfallen kann. Welt und Weltkarte, Original und Kopie, Realität und (kartographische) Fiktion scheinen in eins zu fallen. Dass als 'Quelle' eines solchen Entwurfs ein Reisebericht genannt wird, ist aus der hier gewählten Perspektive keineswegs zufällig. Nicht nur die Karte, auch der Reisebericht hat es 'in sich', ist weit mehr als bloße Abbildung einer Wirklichkeit. Selbst dort, wo als Intention nur das möglichst genaue Verzeichnen von Realität intendiert ist, beobachten wir eine Verselbständigung nicht allein des Vorgefundenen, sondern des Erfundenen.

Stellen wir an diesem Ort eine weitere unserer grundlegenden Fragen, mit der wir an das Verhältnis von Reisen und Schreiben herangehen. Und stellen wir diese Frage auf möglichst einfache Weise so: Wie lässt sich ein Land schreiben, wie lässt sich ein Land beschreiben?

Der französische Schriftsteller und Literaturtheoretiker Michel Butor hat in seiner 'Studie zu einer Repräsentation der Vereinigten Staaten', die 1962 unter dem Titel Mobile ${ }^{17}$ erschien, eine mögliche Antwort auf diese ganz grundsätzliche Frage gegeben. Seiner umfangreichen Abfolge von Kurztexten, die im Gestus des neo-avantgardistischen literarischen Experiments im Querformat abgedruckt wurden, stellt ein Verstehensmodell der USA dar, das sich unter Einbeziehung verschiedenartigster reiseliterarischer Versatzstücke aus unablässigen Bewegungen im Raume wie ein mobiles Puzzle zusammensetzt. Vektorizität ist überall: Alles ist in Bewegung, on the move.

Dabei gibt der Titel des Bandes die möglichen Deutungsrichtungen dieses Bewegungstextes vor. Mobile meint - um nur einige wenige Bedeutungsebenen der Titelsemantik zu nennen - die gleichnamige Stadt im Süden der USA, aber auch den bekannten Konzern, der Treibstoffe für Reisen in unterschiedlichsten Verkehrsmitteln produziert. Der Text selbst ist aber auch ein kunstvolles Mobile, eine Bewegungs-Figur oder Figur der Bewegung, die grundsätzlich nie an ein Ende kommt und immer auch die Bewegungen der Betrachterin oder des Betrachters in sich aufnimmt.

Bewegung steht im Zentrum dieses experimentellen Textes: Alles ist von Vektorizität durchdrungen. Mobile verweist auf die Bewegungen, die von Beginn an das Schaffen des französischen Autors prägten, aber auch auf jenes künstlerische

17 Butor, Michel: Mobile. Etude pour une représentation des Etats-Unis. Paris: Gallimard 1962. 
Artefakt, dessen Einzelteile sich ständig bewegen und damit untereinander wie im Verhältnis zu ihrem Lesepublikum immer wieder neue Konstellationen und Perspektiven ermöglichen. Michel Butors Experimentaltext setzt sich dabei aus einer erstaunlichen Vielzahl von Sprüngen nicht nur zwischen verschiedenen Themen, Geschichtsepochen und Grenzgebieten der USA, sondern - teilweise die Reisebewegungen des Autors autobiographisch aufnehmend - vor allem zwischen einzelnen Bundesstaaten und ihren jeweiligen (Orts-) Namen zusammen. Mobile ist die Versinnbildlichung einer diskontinuierlichen Bewegung: einer Bewegung, die nicht von Kontinuität, sondern von ständigen Sprüngen beherrscht wird.

Aus diesen ungezählten Bewegungen entsteht eine ungeheure Maschinerie, deren unabschließbare Kombinatorik das (Lese-) Publikum in ihr Spiel von und mit Bedeutungen einbezieht und eine Repräsentation der Vereinigten Staaten erlaubt, die nicht räumlich oder zeitlich linear angelegt ist, sondern aus variierenden nicht-linearen Verkettungen besteht. Lineare Bewegungen sind es, die freilich von ständigen Querverbindungen durchtrennt und unterbrochen werden und das Diskontinuierliche veranschaulichen.

Am Anfang dieses Textes, der sich mit Alexander von Humboldt als ein 'netzartig verschlungenes Gewebe' bezeichnen und damit etymologisch korrekt als ein 'Gewobenes', etymologisch als eine 'Textur', verstehen ließe, steht aber eine Landkarte der USA, die einen Überblick über die geographische Lage und die alphabetischen Kürzel der einzelnen Bundesstaaten der Union erlaubt. Erst von dieser kartenschriftlichen Re-Präsentation der Vereinigten Staaten aus entfaltet sich die Mimesis des literarischen Textes als Abfolge diskontinuierlicher Sprünge in Raum und Zeit zugleich. Nichts ist statisch, alles ist beweglich, mobil, in diesem gewobenen Netz, im Netzwerk dieser literarischen Repräsentation, die zugleich einen experimentellen Reisebericht darstellt.

Es ist vielleicht nicht uninteressant, dass Roland Barthes sich in einem nicht in die Essais critiques aufgenommenen Essay sehr intensiv mit Michel Butors Text oder - wenn Sie so wollen - Roman Mobile auseinandergesetzt hat. Doch zuvor möchte ich Ihnen - wie im Rahmen dieser Vorlesung üblich - einige Elemente aus dem Lebenslauf von Michel Butor an die Hand geben.

Michel Marie François Butor wurde am 14. September - am selben Tag wie Alexander von Humboldt, by the way - des Jahres 1926 als viertes Kind des Eisenbahninspektors Emile Butor und seiner Frau Anne Brajeux in Mons-en-Barceul geboren und ist am 24. August 2016 in Contamine-sur-Avre verstorben. Er ist ganz sicherlich der weitestgereiste seiner Schriftstellergeneration: Eigentlich war er beständig auf Achse. Gastaufenthalte und Dozenturen führten ihn nicht nur in verschiedene Städte Frankreichs und Deutschlands, Englands und der Schweiz, sondern auch nach Oberägypten, in die unterschiedlichsten Städte und Universitäten im Norden, Süden und Osten der USA, nach Kanada, aber auch 


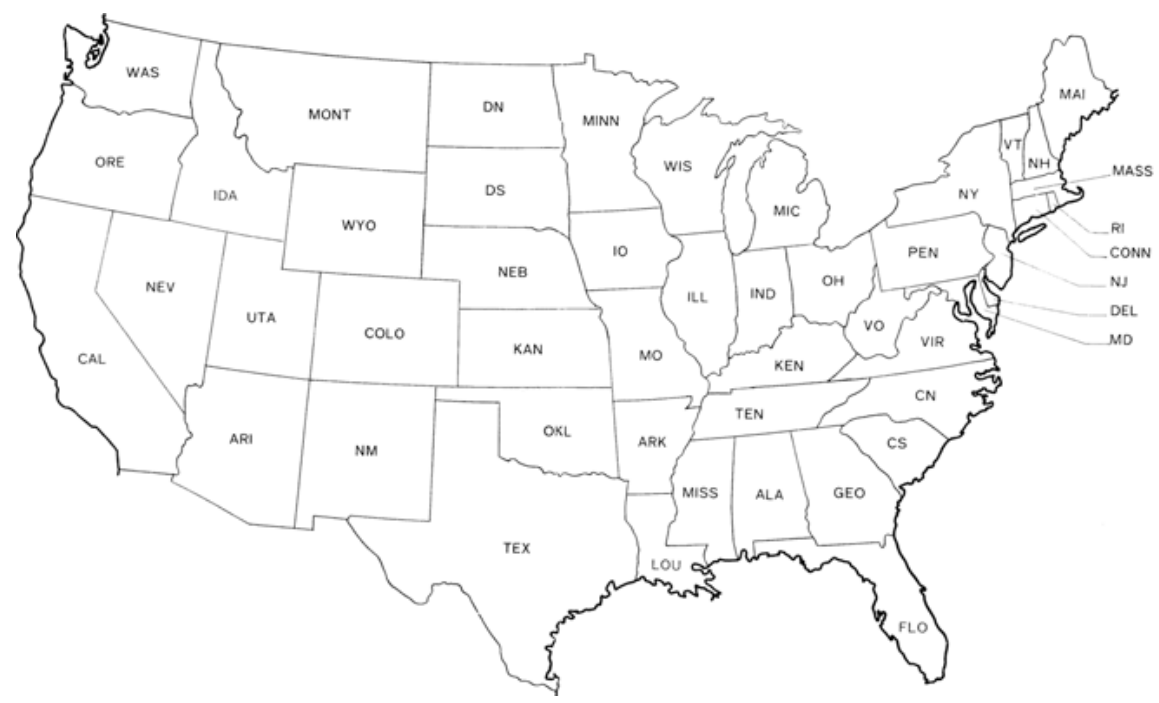

Abb. 19: Karte der USA, abgedruckt in Michel Butors Mobile, 1962.

nach Tunesien und Japan, nach Jugoslawien und Bulgarien, in die Sowjetunion und nach Marokko sowie nach Neuseeland und Australien. Vergessen wir neben allen Reisen nicht, dass er auch ein poeta doctus war, der mit einer Arbeit über den Zusammenhang zwischen Mathematik und Ästhetik promovierte, seit 1970 französische Literatur an der Faculté des Lettres der Universität Nizza unterrichtete sowie seit 1975 einen Lehrstuhl in Genf für moderne französische Literatur innehatte.

Beeinflusst von James Joyce und Marcel Proust, gilt Michel Butor neben Alain Robbe-Grillet und Nathalie Sarraute als bedeutendster Repräsentant des nouveau roman. International bekannt geworden ist Butor seit den sechziger Jahren besonders auch als Essayist ('Repertoire 1-5'). 1971 fand in Frankreich eine bedeutende Tagung zum Thema nouveau roman statt, die fast vollständig dem Werk Butors gewidmet war; 1973 ein internationales Kolloquium über und mit ihm im Centre Culturel International von Cerisy-la-Salle. Michel Butor hat eine Vielzahl von Spuren hinterlassen und gilt als einer der zentralen Autoren der französischen Gegenwartsliteratur. Fügen wir lediglich noch hinzu, dass für Butor der Roman das Laboratorium der Erzählung ist, des récit, der phänomenologische Bereich par excellence: der beste Ort, um herauszufinden, wie die Wirklichkeit auf uns einwirkt und wie wir Wirklichkeit gestalten und erfinden. Ganz klar, dass Michel Butor, der Weitgereiste, ein Erneuerer des literarischen Reiseberichts war. 


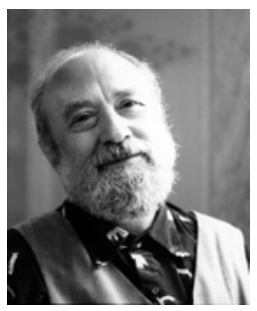

Abb. 20: Michel Butor (Mons-en-Barœul, 1926 - Contamine-sur-Arve, 2016).

Nun aber wieder zurück zu seinem experimentellen Reisebericht aus der französischen Literatur. Michel Butors Mobile aus dem Jahre 1962 ist ein Text seiner eigentlich zweiten Schaffensphase, eine Zeit, die im Grunde in die von Antoine Compagnon genannte Blütezeit der französischen Theoriebildungen fällt. Es sind die großen Jahre französischer Theoriebildung und neoavantgardistischer Schreibpraxis - und unser Autor war zweifellos eine der maßgeblichen Stimmen in diesem neuen und keineswegs unumstrittenen Konzert. Nicht von ungefähr gilt Michel Butor, der über ein mathematisch-literarisches Thema promovierte, zu den sogenannten zerebralen Autoren jener Generation, welche die Nachkriegsliteratur Frankreichs seit den 50er Jahren sehr stark prägte und die man auf Vorschlag eines Kritikers von Le Monde, Emile Herniot, aus dem Jahre 1957 (also dem Jahr von La Modification) zunehmend als nouveau roman bezeichnete.

Freilich: Dass der nouveau roman nun alles andere als eine klar strukturierte, homogene und an ähnlichen Zielen ausgerichtete literarische Bewegung ist, hat uns bereits Roland Barthes sehr deutlich vor Augen geführt. In der Tat haben sich die Hauptvertreter des nouveau roman, also insbesondere Alain Robbe-Grillet, Nathalie Sarraute, Robert Pinget und Michel Butor, stets und wiederholt gegen die gemeinsame Klassifizierung als nouveaux romanciers gewehrt und nicht ohne Koketterie auf ihre so unterschiedlichen Ausrichtungen verwiesen. Das half ihnen aber nichts, denn ihnen wurde - ob sie es wollten, oder nicht - ein Etikett aufgeklebt. Wenn wir nun den Blickwinkel verändern und nicht mehr von der Position des nouveau roman her fragen, was es mit Butors Mobile auf sich hat, sondern vielmehr versuchen, sein Schreiben in den Kontext einer Literatur im Zeichen der Postmoderne zu stellen, verändert sich unsere Fragestellung, aber weitaus mehr noch auch unsere Interpretation und Deutung des Textes - und damit (auf die Differenz zwischen Werk und Text gehe ich noch ein) auch sein Werk selbst. Die spielerische Leidenschaft und jene unbändige Lust, mit den Worten spielen zu können, bleiben freilich bei jeder Perspektivierung erhalten, die sich auf Butor richtet.

Bei Mobile handelt es sich um einen Text, der zum einen unverkennbar als experimenteller Text angelegt ist - und damit ein gewisses avantgardistisches 
Verständnis auch in der Hinsicht projiziert, dass hier ein Bruch mit der literarischen Tradition herbeigeführt werden soll; zum anderen aber geht es auch um einen Text, den man im weitesten Sinne der Reiseliteratur und der Frage einer Alteritätserfahrung zurechnen könnte. Denn es ist ohne jeden Zweifel so, dass die Frage der Alterität und deren Infragestellung gerade für den von uns in der Vorlesung behandelten Zeitraum von größter Bedeutung ist, eine Frage, die letztlich auch die Dimension der Modernen und der vielen Modernen betrifft. Die Erfahrung von Alterität führt die Frage eigener Identität herauf; und genau an diesem Punkt entwickeln sich Vorstellungen von Kultur, die nicht mehr im Zeichen kultureller Homogenität verhandelbar sind.

Michel Butors Mobile ist folglich kein Reisebericht im traditionellen Sinne, sondern ein hochkomplex und zugleich auch hochintellektuell angelegter Text, der - das verschweige ich nicht - vielleicht in den Möglichkeiten, ihn zu denken und neu $\mathrm{zu}$ formieren, anregender ist als in den Möglichkeiten, ihn zu lesen und ihn zu genießen. Doch da kann man durchaus unterschiedlicher Auffassung sein. Der Text ist sicherlich nicht jedermanns Sache - und er will ja auch ganz bewusst seine Leserschaft provozieren.

Ein gut verdauliches Werk über die USA will er ganz bestimmt trotz des etwas reißerischen Klappentextes, der motion und emotion miteinander kombiniert, nicht sein. Diesen Klappentext sollten wir uns aber dennoch einmal anhören, sind es doch sehr oft gerade die Paratexte, die uns den Schlüssel zu einem ansonsten nicht so leicht zugänglichen, vielleicht sogar sperrigen Text liefern. Und schließlich ist das, was auf der Vorder- und Rückseite sowie auf dem Rücken eines Buches abgedruckt ist, ja sehr wohl das einzige, was man bei geschlossenem Buch überhaupt lesen kann und was gerade für die Kaufentscheidung eines Lesers von nicht zu unterschätzender Bedeutung und Wirkung ist. Dieses ist ein Aspekt des Produktes Buch, der Ware Buch, und damit sein Zusammenhang mit der Konsumwelt, ja mit dem Massenkonsum in einer kapitalistischen Warengesellschaft. Und diese Ware Buch wird hier sehr wohl angepriesen:

Respirez l'air des 50 états!

De ville en ville, de frontière en frontière, de la côte Atlantique à la côte Pacifique!

Des centaines de fleuves, des centaines d'oiseaux, des centaines de voix! Les Européens, les Noirs, les Indiens!

Vivez aujourd'hui avec votre famille la rigolade, l'aventure, le drame du passé, du présent et du futur de l'Amérique!

Voyagez à travers un continent, à travers des siècles, pour jouir des frissons d'un spectacle grand comme l'Amérique elle-même!

Excitation! Aventure! Education! 
Depuis la Nouvelle Angleterre coloniale jusqu'à l'Ouest des pionniers, d e la frontière Mexicaine aux ports des Grands Lacs, du Cap Canaveral au passage du Nord-Ouest!

Feuilletez les ouvrages du grand peintre et naturaliste John James Audubon, lisez les déclarations du président Jefferson, et suivez un véritable procès de sorcière!

Regardez les américains, vivez avec les américains, roulez dans leurs longues voitures, survolez leurs aérodromes, déchiffrez leurs enseignes lumineuses, flânez dans leurs grands magasins, plongez-vous dans leurs immenses catalogues, étudiez leurs prospectus, arpentez leurs rues, dormez sur leurs plages, rêvez dans leurs lits!

Mobile!

Une orgie de surprises et de frissons! $!^{18}$

[Atmen Sie die Luft von 50 Staaten!

Von Stadt zu Stadt, von Grenze zu Grenze, von der Atlantik- bis zur Pazifikküste!

Hunderte von Flüssen, hunderte von Vögeln, hunderte von Stimmen! Die Europäer, die Schwarzen, die Indianer!

Leben Sie mit Ihrer Familie heute den Spaß, das Abenteuer, das Drama der Vergangenheit, der Gegenwart und der Zukunft Amerikas!

Reisen Sie durch einen Kontinent, durch Jahrhunderte, um den Nervenkitzel einer Show so groß wie Amerika selbst zu genießen!

Aufregung! Abenteuer! Bildung!

Vom kolonialen New England bis zum Westen der Pioniere, von der mexikanische Grenze bis zu den Häfen der Großen Seen, von Cape Canaveral bis zur Nordwestpassage!

Durchblättern Sie die Werke des großen Malers und Naturforschers John James Audubon, lesen Sie die Äußerungen Präsident Jeffersons, und folgen einem echten Hexenprozess!

Schauen Sie sich die Amerikaner an, leben Sie mit den Amerikanern, fahren Sie in ihren langen Autos, überfliegen Sie ihren Flugplätze, entschlüsseln Sie ihren Neonleuchtschilder, flanieren Sie durch ihre Kaufhäuser, tauchen Sie in ihre umfangreichen Kataloge ein, studieren Sie ihre Prospekte, gehen ihre Straßen, schlafen an ihren Stränden, träumen in ihren Betten!

Mobile!

Eine Orgie von Überraschungen und Nervenkitzel!]

Wir haben es hier mit einem im besten Sinne marktschreierischen Text zu tun. Es ist ein Stückchen Reiseliteratur, das zum Reisen auffordert, zu einem Mitreisen, das sich vermeintlich mitreißend gestaltet. In jedem Falle aber ist es ein Stück Literatur, das im Reiseakt selbst seinen eigentlichen Beweg-Grund erblickt und eine invitation au voyage an seine Leserschaft ausspricht. Der Band verspricht, nicht nur zu einer Lesereise einzuladen, sondern auch viele Leseabenteuer erleben zu lassen. Folgen wir also dieser herzhaften Einladung und brechen wir mithin zu einer Reise ins Unbekannte auf.

Liest man die Biographie Michel Butors, so weiß man, wie sehr er selbst von Reisen fasziniert war. Schon bald nach dem Krieg sieht man ihn auf

18 Butor, Michel: Mobile. Etude pour une représentation des États-Unis. Paris: Gallimard 1992, Klappentext. 
Reisen in den unterschiedlichsten Ländern Europas, bald aber auch weltweit, wobei den Aufenthalten nicht zuletzt auch an nordamerikanischen Universitäten gerade in den Jahren vor der Veröffentlichung von Mobile eine große Bedeutung zukommt. Butor liebte es, immer wieder neue Reiseerfahrungen zu machen und zugleich sich seine Reiseerlebnisse überlagern zu lassen. Auch noch der alte Michel Butor hat seine planetarischen Erfahrungen stets miteinander verwoben und ein transareales Erdgefühl entwickelt. Hatte er nicht wie Alexander von Humboldt an einem 14. September Geburtstag? Nicht umsonst prägt viele der Butor'schen Texte ein ausgesprochenes Reisefieber im konkreten wie im übertragenen Sinne.

Der oben angeführte Text nun zitiert ein solches Reisefieber herbei. Er ist vergleichbar mit dem zumindest französischen Stil der Werbung für Reisen, der sich durchaus von deutschen Standardtexten für Reisewerbung unterscheidet. Insoweit handelt es sich wirklich um einen Werbetext von der Gattung her, der im Übrigen zugleich eine ironische Distanz aufbaut zu einer Funktion, die er gleichzeitig erfüllt: nämlich für das Buch, als dessen Klappentext er fungiert, entschlossen zu werben.

Der Klappentext von Mobile ist also ein appetizer, der seine Wirkung durchaus entfalten kann, macht er doch zumindest auf dieses Buch großen Appetit. Wir sehen bereits hier den von Barthes angesprochenen notwendigen und unhintergehbaren Zusammenhang auch noch der subtilsten Literatur, die sich an eine Öffentlichkeit wendet, mit den Mechanismen, Strategien und Bedingungen der Massenkommunikation - gerade auch in einem Reisebuch, das in den Zeit des Beginns des Massentourismus fällt. Butor wählt hier auf dem Klappentext die Strategie der Übersteigerung, die eine ironische Distanzierung mit sich bringt und damit in sich selbst die Problematik der Verbindung zwischen hoher Literatur und Massenkonsum und Massenkommunikation präsentiert und integriert. Dabei dürfen wir die Position des Autors gegenüber seinem Werbetext wohl zweifellos als ironisch beschreiben.

Zugleich suggeriert dieser Text natürlich, dass man durch die Lektüre dieses Buches selbst eine Reise unternehmen und Abenteuer erleben kann. Diese invitation au voyage macht darauf aufmerksam, dass das Lesen selbst eine Reise ist, angefangen mit der Bewegung der Augen auf dem Papier, die eine Reise des Verstehens ist. Dies hatten wir schon gleich zu Beginn unserer Beschäftigung mit der Reiseliteratur gesehen.

Damit aber ist die Reisemetaphorik eingeschrieben in die Grundstruktur des Textes selbst. Es ist eine bekannte Tatsache, dass sich die Metaphorik der Reise von Beginn der Romangeschichte, also der Geschichte der Gattung des Romans, prominent findet. Die Reise nimmt eine zentrale Stellung ein bei der Erklärung der Struktur von Cervantes’ Don Quijote bis hin zu A la recherche $d u$ 
temps perdu, vom Lazarillo de Tormes und dem vorgelagerten Abenteuerroman und Roman de geste bis hin zu den aktuellsten Thrillern unserer Tage. Selbstverständlich beschränkt sich die Reisemetaphorik als Grundstruktur nicht auf den Roman, findet sich in anderen Texten - denken Sie an die Comedia, die Göttliche Komödie Dantes - bis hin zum Selbstverständnis und zur Selbstversicherung unseres Lebens selbst, das natürlich ebenfalls als Reise, und sei es als eine Pilgerreise, angesehen werden kann.

Sicherlich ist im übertragenen Sinne jede Lektüre eines Textes eine Reise: von den ersten Buchstaben auf dem Umschlag bis hin zum Ende der Schrift, wenn wir eine lineare Lektüre praktizieren. Die Reisestruktur ist damit eine anthropologische Konstante, zugleich aber eingeschrieben in die Gattung des Romans, nicht zuletzt aber natürlich auch des literarischen Reiseberichts, das versteht sich von selbst. Was sich uns hier ankündigt, ist nun eine Reise der besonderen Art, eine Reise durch verschiedene Regionen eines Kontinents, eine Reise durch verschiedene Zeiten, eine Reise durch die verschiedenen Ethnien, die diesen Kontinent bevölkern, aber auch eine Reise durch verschiedene Daseinsformen, die sich in dieser Welt namens Amerika ein Stelldichein gegeben haben. Amerika erscheint als das Andere Europas, steht im Zeichen einer fundamentalen Differenz, die alle Bereiche des Lebens erfasst.

Doch ziehen wir an dieser Stelle mit Blick auf unsere Ausgangsfrage schon einmal eine Art Zwischenbilanz. Die experimentellen Texte von Jorge Luis Borges, Adolfo Bioy Casares und Michel Butor führen zwei mögliche Antworten auf die Frage vor, wie sich ein Land schreiben, beschreiben und repräsentieren lässt. Diese Antworten sind keineswegs erschöpfend, deuten aber zwei grundlegende Richtungen der Beantwortung an.

Bemerkenswert ist, dass ihre 'Lösungen' von der kartographischen Repräsentation ausgehen, die im System abendländischer Darstellungsmodi mit Blick auf ihre Wirklichkeitstreue wie auf ihre pragmatische Verwertbarkeit höchste Wertschätzung genießen. Mit Hilfe von Karten wird ein Raum überschaubar, übersichtlich und daher analysierbar, von der Vernunft zerlegbar gemacht: Er kann immer stärker untergliedert und immer detaillierter präsentiert werden, ohne dass der Bezug zur Wirklichkeit außerhalb des Kartennetzes gekappt würde. Michel Butors Buch ist nicht von ungefähr eine Karte der USA vorangestellt. Die kartographische Darstellung vermittelt die Kontinuität eines Raumes, der durch seine Grenzen - wie bei den Grenzen eines Reiches - markiert wird und als eine Einheit erscheint, deren territoriale Kontinuität sich der Wirklichkeit auflagert.

Im Unterschied zu der von Borges und Bioy Casares vorgeführten Lösung, in der die Repräsentation in Wirklichkeit, das Fiktionale in das Faktische umkippt, gerade weil die Kontinuität und Einheit einer Fläche suggeriert wird, wendet sich das Textmobile Butors einer nicht-kontinuierlichen Darstellungsweise zu. Deren 
Sprünge in Raum und Zeit, zwischen unterschiedlichen Lebewesen und Lebensformen werden nur dadurch ermöglicht, dass zuvor vermittels einer kartographischen Darstellung die Grundlage für eine derartige Kombinatorik und Relationalität geschaffen wurde. der Weg durch die USA ist in Mobile gerade nicht kontinuierlich und itinerarisch, sondern stellt ein Hüpfen quer über den Teilkontinent dar.

Es gäbe viele Möglichkeiten, die große Aktualität und Bedeutung der Reiseliteratur für die Literaturen der Welt insgesamt herauszuarbeiten. Vom Don Quijote war schon die Rede; und jeder Leser dieses ersten europäischen Romans der Moderne weiß, dass ihm ständige Reisebewegungen als Bewegungen in Raum und Zeit zu Grunde liegen, auch wenn das Fortbewegungsmittel, die Klappermähre Rocinante, nicht gerade mit hoher Geschwindigkeit gesegnet ist. Wir sollten dabei nicht vergessen, dass Cervantes bei seiner Schaffung des modernen Romans auf das Genre der Novela picaresca, des Schelmenromans zurückgriff, der seinerseits eine Abfolge von Reisen darstellt, von inkohärenten Reisebewegungen des Protagonisten oder der Protagonistin, welche als Grundstruktur ebenso auf den Abenteuerroman als auf den Reisebericht zurückverweisen. So ist im Kern der großen abendländischen Gattung des Romans jenseits aller Bewegungen, die natürlich den Akt des Lesens selbst auch betreffen, die Reiseliteratur auf fundamentale Weise eingeschrieben.

Lassen Sie mich an diesem wichtigen Punkt unserer Vorlesung noch auf eine zweite Dimension hinweisen, die auf die unbedingte Aktualität und den veränderten Kontext von Reiseliteratur heute aufmerksam machen. Denn es gibt eine weitere Gattung oder Textsorte, die heute von äußerster Aktualität ist und eminente Bezüge zur Reiseliteratur aufweist. Ich spreche von dem, was häufig als Migrationsliteratur bezeichnet wird, die ich an dieser Stelle aber als eine Literatur ohne festen Wohnsitz benennen möchte, jener Literatur also, die aus der Erfahrung der Deplatzierung und Migration über die Migration und Deplatzierung schreibt, um einmal eine etwas enger gefasste Definition heranzuziehen. Diese Literatur, die Teil der Literaturen ohne festen Wohnsitz ist, ist in vielerlei Hinsicht die Darstellung einer Alteritätserfahrung, die ebenfalls im Zentrum der Reiseliteratur steht, nur dass die Bewegungsfiguren im hermeneutischen Sinne andere sind und eine Reise mit Rückkehr eine grundsätzlich andere Verstehensstruktur evoziert als umgekehrt die Darstellung von Bewegungen und Migrationen, die sich vielleicht ohne die Möglichkeit einer Rückkehr vollziehen. Über lange Zeit war für diese Literatur auch die Bezeichnung 'Migrantenliteratur' zu hören, eine letztlich sehr pejorative Bezeichnung, mit der wir im Rahmen dieser Vorlesung nichts zu tun haben wollen, da sie eine ausschließende Denomination darstellt.

Als ein kleines Beispiel für eine derartige Literatur ohne festen Wohnsitz möchte ich gerne auf die deutsch-türkische Autorin Emine Sevgi Özdamar 
hinweisen, allerdings nicht - wie an anderer Stelle - auf ihren Erzählband Mutterzunge, sondern auf jenen Roman, für den sie 1991 mit dem Ingeborg-BachmannPreis in Klagenfurt ausgezeichnet wurde. Ihr aufsehenerregender Roman erschien dann im Folgejahr 1992 unter dem schönen Titel Das Leben ist eine Karawanserei hat zwei Türen aus einer kam ich rein aus der anderen ging ich raus. Jahre später avancierte dieser Roman dann zum Eröffnungstext einer Trilogie, die im Spannungsfeld von Berlistan spielt: also irgendwo zwischen Istanbul und Berlin in einem ständigen Bewegungsfeld, das beide Städte intim miteinander verbindet.

Emine Sevgi Özdamar, die 1946 in Malatya in Anatolien geborene Türkin, die von 1965 bis 1967 erstmals nach Deutschland, nämlich als Fabrikarbeiterin nach Westberlin kam, ist spätestens seit Anfang der 90er Jahre eine der wichtigsten Stimmen nicht nur der deutschsprachigen Literatur an der Wende zum 21. Jahrhundert. Ihre Regie- und Theaterarbeit an der Ostberliner Volksbühne, später dann in Paris und am Schauspielhaus Bochum führten sie zur Literatur, zum Schreiben in deutscher Sprache; und es verwundert nicht, dass angesichts dieser ständigen räumlichen Veränderungen, von denen hier nur die wichtigsten genannt wurden, sich eine Literatur ohne festen Wohnsitz entwickelte, welche ein translinguales Schreiben nicht in der 'Mutterzunge', sondern in der zunächst fremden Sprache des Deutschen als Grundlage hat. Ja, es ist eine Literatur translingualen Zuschnitts, die sich des Deutschen auf eine sehr eigentümliche Art und Weise bedient. Was ist darunter zu verstehen?

Beschäftigen wir uns also kurz mit Emine Sevgi Özdamars Eröffnungsroman der Trilogie. Das Leben ist eine Karawanserei erzählt fast ausschließlich im Sinne einer Autofiction - also eines autobiographischen Schreibens ohne autobiographischen Pakt - von Kindheit und Jugend in der Türkei, die auf Grund der schwierigen Arbeitssituation des Vaters von ständigen Ortswechseln geprägt waren: Von Malatya im Kurdenland nach Istanbul, von Istanbul nach Bursa, nach Ankara und innerhalb der jeweiligen Orte - ständig von Armut bedroht -, bevor schließlich der Weg erneut nach Istanbul, bevor endlich von dort der Weg in einer denkwürdigen Ausreise nach Deutschland, nach Westberlin, führt. Es ist eine Migrationsgeschichte, genauer: eine Arbeitsmigrationsgeschichte, wie sie millionenfach zu erzählen war und ist.

Laut Klappentext der aktuellen Ausgabe ${ }^{19}$ handelt es sich dabei um „eine Art orientalischen Bildungsroman“ und zugleich um ein „europäisches Werk des magischen Realismus“ - marktgerechte Formulierungen, die ihre Wirkung nicht verfehlten und doch dem Romangeschehen und seiner literarischen Präsentation

19 Özdamar, Emine Sevgi: Das Leben ist eine Karawanserei - hat zwei Türen - aus einer kam ich rein aus der anderen ging ich raus. Köln: Kiepenheuer\&Witsch 1994. 


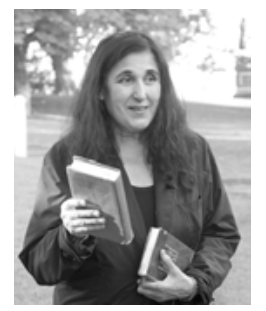

Abb. 21: Emine Sevgi Özdamar (Malatya, 1946).

nicht gerecht werden. Gerade die ostentative Verknüpfung mit dem 'magischen Realismus' lateinamerikanischer Prägung sollte uns ein wenig stutzig machen, denn sie leitet sich zwar ursprünglich aus der europäischen Kunstgeschichte ab, wurde dann aber auf eine bestimmte Schreibweise des hispanoamerikanischen Romans bezogen, der als Realismo mágico - ausgehend von Miguel Angel Asturias über Gabriel García Márquez bis hin zu Isabel Allende - bezeichnet wurde. Das war gleichsam das Erfolgsrezept großer Autoren aus Lateinamerika - und damit hat der Roman von Emine Sevgi Özdamar wirklich nicht viel gemein. Denn er geht seine eigenen Wege und benutzt auch eine Sprache, die mit jener des 'magischen Realismus' keinerlei Ähnlichkeit besitzt. Es ist also eine Werbebotschaft, auf deren Werbung wir nicht hereinfallen sollten.

Gleichwohl kommt damit paratextuell eine gleichsam außereuropäische Färbung in die Rezeption dieses Romans, der letztlich die Vorstufe einer Entwicklung schildert, die zur Auswanderung nach Europa oder - aus anderer Perspektive - zur Arbeitsmigration nach Westberlin führte. Ein Gutteil des Lebens wird damit gleichsam zu einer Art Reisebericht - wie es der Titel schon sagt: Das Leben ist eine Karawanserai. Um was für eine Reise geht es hier aber? Und inwiefern ist sie charakteristisch für das, was die Flüchtlingswellen des 21. Jahrhunderts in verschiedenen Ländern Europas bewirkt haben?

Ich möchte Ihnen an dieser Stelle wenigstens in aller Kürze einen kleinen Einblick in die Grundstruktur dieses Romans geben anhand einer Passage, die sich auf den Augenblick der Abreise, der Abfahrt - einen, wie wir noch sehen werden, wichtigen reiseliterarischen Ort - bezieht. Auch hier wird mit dem Reisen das Lesen gekoppelt, freilich auf eine etwas andere Art und Weise. Wir befinden uns im Jahr 1965:

Mutter nahm einen Stein in die Hand und sagte: 'Schau, bis du wiederkommst, werde ich einen Stein auf meine Brust drücken.'

Ich stieg in den Zug nach Deutschland ein, auch viele andere Frauen stiegen ein. Es gab nur einen einzigen Mann, der einstieg, es war der Zugleiter. Er verteilte an uns einen Plastikkrug mit Wasser, ein Paket Essen, 112 DM, die ein Teil unseres Monatslohns waren, und ein Buch. Das Buch hieß: 'Handbuch für die Arbeiter, die in der Fremde arbeiten gehen.' [. . . ] 
Die Universitätsschülerin las weiter: 'In Europa trägt man kein Kopftuch. Wenn türkische Frauen ein Kopftuch tragen, wird Europa sie nicht lieben. Bitte, liebe Schwester, Arbeiterin, trage kein Kopftuch. Wenn es unbedingt sein soll, tragt es bitte so, wie europäische Frauen Kopftücher tragen.'

Der Zug fuhr noch lange sehr dicht an den alten Istanbuler Häusern vorbei. Drinnen saßen alte Menschen und schauten auf den Zug, als ob er ein kleiner, gewöhnlicher Wind für sie wäre. ${ }^{20}$

Soweit diese kurze Passage aus den letzten Seiten des Romans, die uns den Abschied und die sich abzeichnende Auseinandersetzung der jungen ausreisewilligen Türkinnen mit einer anderen Gesellschaft 'in der Fremde' skizzieren. Am Ende des Romans öffnet sich das Geschehen also auf Berlin, genauer: auf Westberlin, wohin die Frauen gebracht oder eigentlich verfrachtet werden. Wie gesagt: Wir wohnen einer Szenerie bei, wie sie sich millionenfach in der Türkei, aber auch anderswo abgespielt hat. Was macht aber die individuelle Prägnanz und den Fokus dieser Szene aus? Und was kennzeichnet diesen Roman, der für eine ganze Reihe von deutsch-türkischen Schriftstellern zum vielkopierten Modell wurde?

Es wird in dieser Szenerie deutlich, dass die Arbeitsmigration, mit Hilfe derer die Protagonistin der Armut und dem Hunger in ihrer Familie und ihrer Heimat entfliehen will, zugleich ein Abschied von der Mutter, aber auch von der Mutterzunge, ein Abschied vom Alten, aber auch von den althergebrachten, tradierten Kulturformen ist. Der den Arbeiterinnen mitgegebene Leitfaden soll möglichst eine rasche Assimilierung der jungen Türkinnen an das 'Gastland' hervorrufen, wobei die eigenkulturellen Prägungen am Beispiel des Kopftuches möglichst verschwiegen oder zumindest kaschiert werden sollen. Elemente des islamischen Glaubens der jungen Türkinnen sollen möglichst komplett zum Verschwinden gebracht und nur mehr ihre Arbeitskraft in den Mittelpunkt gerückt werden. Mit der Deplatzierung von Istanbul nach Berlin geht somit eine kulturelle Assimilierung einher, die sich schon in diesem Romanerstling andeutet, aber dann in den weiteren Fortsetzungsromanen ausgeführt wird. Denn was wirklich geschieht, ist eine transkulturelle Erfahrung zwischen der Türkei und Deutschland, zwischen Istanbul und Berlin, die das Oszillieren zwischen den beiden großen Städten in ein eigentümliches Bewegungsmuster verwandelt. Man könnte sagen, dass auf diese Weise ein transkulturelles Berlistan entsteht, in welchem wir es weder mit Istanbul noch mit Berlin allein zu tun haben. ein sich wie der Zug erst langsam beschleunigender Prozess der Querung zumindest zweier Kulturen beginnt.

Emine Sevgi Özdamars Roman war bereits vor dieser Schlussszene des Aufbruchs ins ferne Deutschland ein ständiger Reisebericht, ein Bericht von einer ununterbrochenen Karawanserei, gewesen. Denn die komplexe Binnenmigration 
der Familie der Protagonistin geht allen anderen Bewegungen und vor allem der transnationalen Außenmigration voraus. Nicht umsonst hatten alle Bewegungen, die den Roman durchpulsen, gleich in der Eingangsszene begonnen. Auch dies war eine Szenerie aus einem Zug - ganz symmetrisch also der Aufbau des Romans. An diese Szene erinnert sich die Protagonistin und Ich-Erzählerin noch aus der Perspektive des Bauches ihrer Mutter, also vor ihrer eigenen Geburt: Schon zum damaligen Zeitpunkt war sie eine Migrantin, eine von einem zu vielen anderen Orten Reisende gewesen - ohne jeden festen Wohnsitz. Im Grunde hat sich für sie nichts Wesentliches verändert: Sie lebt in einer Fremde, die immer eine andere ist und quer zu den Kulturen verläuft.

Die symmetrische Struktur des Romans zeigt sich auch noch auf einer anderen Ebene: Die Mutter - und mit ihr auch die Mutterzunge - steht zu Beginn und am Ende von Das Leben ist eine Karawanserai, der von ständigen Bewegungen geprägt ist, wobei die hier geschilderte am Ende des Textes zugleich die erste ist, zu der sich die Ich-Erzählerin selbständig entschieden hat. Ihr Entschluss, die Türkei zu verlassen, eröffnet im Grunde erst die Möglichkeiten der Folgeromane. Alle anderen Bewegungen, die wir im Fortgang des Romans durchlaufen haben, sind für die Protagonistin freilich nicht vorüber: Sie sind gleichsam wie in einem Vektor aufgehoben und noch immer enthalten, bedingen damit auch die künftigen Bewegungen der aktuellen wie der künftigen Reisen und Migrationen wesentlich mit. Dies veranschaulicht, wie Vektorizität funktioniert: Sowohl vergangene als auch künftige Bewegungen sind in der Vektorizität beispielsweise eines Romans gespeichert, wobei diese Vektorizität alle möglichen Bewegungen einspeichern und wieder zum Vorschein bringen kann oder uns etwas darüber aussagt, wie eine künftige Bewegung verlaufen kann. Bewegung steht im Herzen der Reiseliteratur. 